\title{
PROPUESTA METODOLÓGICA PARA ESTIMACIÓN DE LA OFERTA Y DEMANDA HÍDRICA EN UN DISTRITO DE RIEGO
}

\section{Methodological proposal for the estimation of water supply and demand in a irrigation distric}

\author{
Alejandro Franco Rojas ${ }^{1}$;; María Trochez Páez ${ }^{2}$ \\ ${ }^{1}$ Docente, Universidad de La Salle. Email: afrancor@unisalle.edu.co \\ ${ }^{2}$ Ingeniera Ambiental y Sanitaria, Universidad de la Salle. Email: mariatrochez29@gmail.com
}

\section{Resumen}

El rendimiento hídrico de Colombia $\left(56\right.$ 1/s* $\left.\mathrm{km}^{2}\right)$ supera ampliamente el promedio mundial (10 $1 / \mathrm{s}^{*} \mathrm{~km}^{2}$ ), pero la sobreexplotación amenaza su sostenibilidad, especialmente porque la demanda de agua para agricultura representa el $46,2 \%$ del total nacional (ENA, 2014) y determinar el agua para riego suele ser altamente empírico. Consecuentemente, la Ley 373/1997 obliga a todo usuario del recurso hídrico, presentar el Programa de Uso Eficiente y Ahorro del Agua (PUEAA) ante la autoridad ambiental, constituyendo el balance entre oferta hídrica y demanda un insumo necesario.

Buscando formular una metodología para dicho balance, en condiciones de déficit de información, se adoptó como caso de estudio el distrito de riego ASOAT-ROCA ubicado en Ubaque, Cundinamarca, este distrito surte 161 usuarios, con una concesión de 60L/seg del río Palmar otorgada por Corporinoquia.

Se adoptó la ecuación de Blanney Criddle para estimar la evapotranspiración de cultivo y se realizó un análisis hidrológico regional para cuantificar la oferta de agua de la fuente abastecedora. Por tanto, los insumos fueron un DEM y registros históricos del IDEAM para estaciones de cuencas vecinas.
Para caracterizar la demanda, se aplicaron 27 entrevistas semiestructuradas sobre tipos de cultivos, métodos de riego y prácticas ambientales.

Se logró cuantificar el requerimiento de agua (ETo) de cada cultivo, el aporte por precipitación y el caudal teórico de riego (ETo-P), que se comparó con la oferta de año seco $\left(\mathrm{Q}_{95}\right)$ y el consumo real según macromedición. Los resultados permiten concluir que la captación para riego no constituye un riesgo para el sostenimiento de la oferta.

Palabras clave: agricultura, evapotranspiración, sostenibilidad, uso eficiente

\begin{abstract}
The water yield of Colombia $\left(561 / \mathrm{s}^{*} \mathrm{~km}^{2}\right)$ far exceeds the world average $\left(101 / \mathrm{s}^{*} \mathrm{~km}^{2}\right)$, but overexploitation threatens its sustainability, especially because the demand for water for agriculture represents $46.2 \%$ of the national total (ENA, 2014) and determining water for irrigation is usually highly empirical. Consequently, Law $373 / 1997$ requires all users of water resources, to present the Efficient Use and Water Saving Program (PUEAA) before the environmental
\end{abstract}


authority, constituting the balance between water supply and demand a necessary input.

Looking to formulate a methodology for this balance, in conditions of information deficit, the irrigation district ASOAT-ROCA located in Ubaque, Cundinamarca, was adopted as a case study. This district has 161 users, with a concession of $60 \mathrm{~L} / \mathrm{sec}$ of the Palmar river granted by Corporinoquia.

The Blaney Criddle equation was adopted to estimate crop evapotranspiration and regional hydrological analysis was carried out to quantify the supply of water from the supply source. Therefore, the inputs were a DEM and historical records of the IDEAM for stations in neighboring basins.
To characterize the demand, 27 semi-structured interviews were applied on crop types, irrigation methods and environmental practices.

It was possible to quantify the water requirement (ETo) of each crop, the contribution by precipitation and the theoretical flow of irrigation (ETo-P), which was compared with the supply of dry year $\left(\mathrm{Q}_{95}\right)$ and the actual consumption according to macromeasurement. The results allow to conclude that the collection for irrigation does not constitute a risk for the sustainability of the supply.

Keywords: Agriculture, evapotranspiration, sustainability, efficient use

\section{Introducción}

La Ley 373 de 1997 estípula que cualquier empresa prestadora de servicios de acueducto, alcantarillado, riego y drenaje que haga uso del recurso hídrico debe presentar el Programa de Uso Eficiente y Ahorro del Agua (PUEAA), que incluye el conjunto de estrategias y programas a los que se compromete el usuario para cumplir con los objetivos fundamentales de uso adecuado del recurso y reducción de pérdidas. Es así, que la cuantificación de la oferta hídrica de las fuentes de abastecimiento y la demanda de los usuarios, constituyen insumos indispensables para su formulación; sin embargo las restricciones para la obtención de información hidrometeorológica por ausencia de estaciones así como de volumen de consumo a causa de la no sistematización de la información por parte de los usuarios, limitan la correcta valoración de la oferta y demanda hídrica, dificultando la ejecución de medidas acertadas para la protección y sustentabilidad del recurso hídrico.
Como caso de estudio, se presenta la Asociación del Distrito de Riego de Adecuación de Tierras de Pequeña escala de Romero y Centro Afuera (ASODAT-ROCA) ubicado en Ubaque, Cundinamarca. Este distrito, cuenta con concesión de aguas de 60L/seg del río Palmar, otorgada por Corporinoquia, para surtir a 161 usuarios y regar 101 ha, distribuidas así: habichuela (32,65 ha), tomate $(9,04$ ha), pimentón (8,08 ha), maíz (6,09 ha), girasol (4,61 ha), tomillo (13,84 ha) y café $(26,30$ ha).

Para segura obtener información de cada uno de los cultivos arriba descritos, se seleccionó una muestra representativa de usuarios, con esto, se cuantificó la demanda de uso agrícola siguiendo la ecuación de Blanney Criddle. Considerando la inexistencia de estaciones medidoras de caudal en el río Palmar, se recurrió a registros de estaciones localizadas en cuencas vecinas aplicando la metodología de rendimiento hídrico. Como resultado se estimó el consumo de agua para riego el cual se comparó con los registros de macromedición y con los caudales para condiciones hidrológicas medias y secas, para 
61

finalmente formular estrategias encaminadas al uso eficiente y ahorro del agua, cuya implementación aporta a la sostenibilidad del recurso y el crecimiento económico del distrito.

\section{Metodología}

Inicialmente se delimitó la cuenca mediante herramientas SIG y se recopilaron registros de caudales diarios suministrados por el IDEAM de las estaciones hidrometeorológicas cercanas a la fuente abastecedora: Oro Podrido (35027020), Guacapate (35027190), Llano Largo (35027220) y Caraza (35027100) (ver Figura 1).

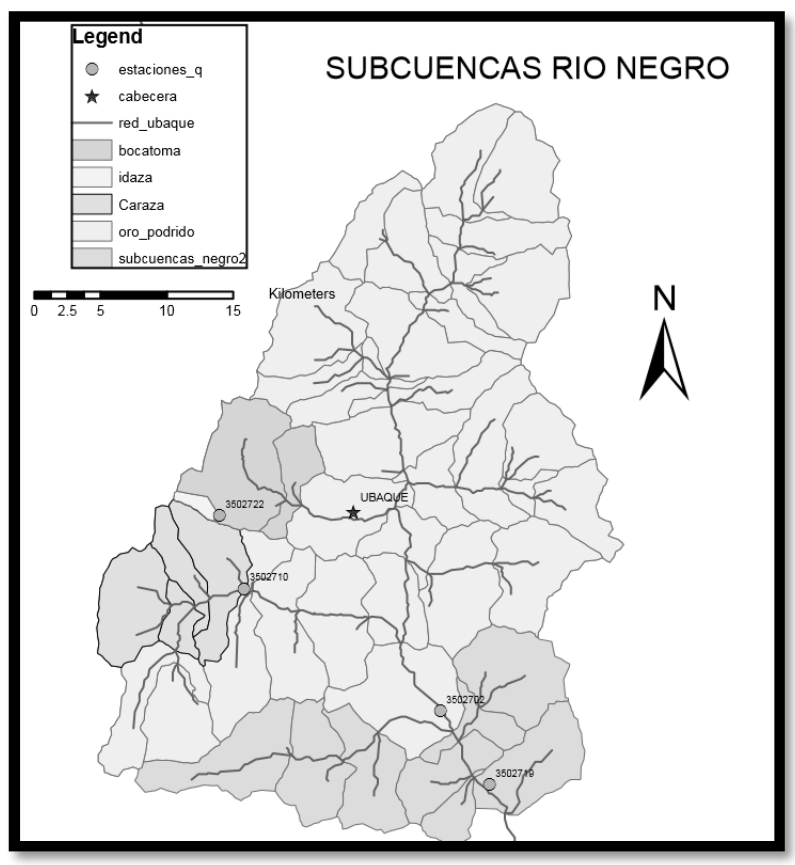

Figura 1. Delimitación de la cuenca y localización de estaciones hidrometeorológicas

Para caracterizar la demanda, del total de suscriptores se seleccionó una muestra de 27 usuarios a quienes se les aplico una entrevista semiestructurada que incluyó cuatro secciones: Información general del usuario, descripción de cultivos, métodos de riego y buenas prácticas ambientales; está información fue sistematizada en una base de datos.
Para cuantificar la oferta hídrica del río Palmar, se determinó el área afluente a cada estación hidrológica utilizando la herramienta Hydrology del programa Arcgis y el modelo digital de elevación de terreno (DEM). Se construyó la curva de duración para cada estación, y al dividir los caudales característicos $\left(\mathrm{Q}_{\mathrm{Max}}, \mathrm{Q}_{95}, \mathrm{Q}_{50}, \mathrm{Q}_{5} \mathrm{y}\right.$ $\mathrm{Q}_{\mathrm{Min}}$ ) por el área respectiva, se obtuvo el rendimiento hídrico $\left(\mathrm{Lt} / \mathrm{seg}^{*} \mathrm{~km}^{2}\right)$, de tal forma que al multiplicar este rendimiento por el área de drenaje del río Palmar se determinó el régimen de caudales en el punto de la bocatoma.

La demanda de uso agrícola (ETo) fue calculada según información aportada por los usuarios en las entrevistas, información que fue incorporada en la ecuación de Blanney Criddle, especialmente en lo relacionado con las etapas de desarrollo y duración de los ciclos vegetativos. Por último se construyó la curva del coeficiente del cultivo Kc para tomillo, tomate, maíz, habichuela, pimentón y girasol, tomando como referencia los valores reportados por Aparicio (1989) y FAO (2006). Teniendo en cuenta registros de precipitación (P) y la demanda de uso agrícola (ETo), para cada mes se obtiene el requerimiento de riego (ETo-P), datos que posteriormente son graficados con el fin de comparar la oferta hídrica del río Palmar en condiciones climáticas secas $\left(\mathrm{Q}_{95}\right)$ respecto a la demanda de uso agrícola. A partir de este análisis se realizó un diagnóstico ambiental sobre el uso del agua por parte de ASODAT-ROCA, que condujo a la formulación de estrategias y acciones dirigidas al cumplimiento de tres aspectos: el uso eficiente del agua, la conservación y protección de nacimientos y áreas estratégicas, así como la educación ambiental, bases para el posterior desarrollo del PUEAA.

\section{Resultados}

El área total objeto de riego varía entre 59,97 Ha y 101,60 Ha, en los meses de julio y mayo, respectivamente. Los cultivos con mayor requerimiento de agua por evapotranspiración son la habichuela $(26,84 \mathrm{Lt} / \mathrm{s})$ y el café $(13,81 \mathrm{Lt} / \mathrm{s})$, 
62

mientras que el resto de cultivos tienen un requerimiento inferior a los $4 \mathrm{Lt} / \mathrm{s}$, cada uno (ver Tabla 1).

\begin{tabular}{|c|c|c|c|c|c|c|c|c|}
\hline \multirow{2}{*}{ Mes } & \multicolumn{7}{|c|}{ Requerimiento hídrico (Lt/s) } & \multirow{2}{*}{$\begin{array}{c}\text { Área } \\
\text { cultivo } \\
\text { (ha) }\end{array}$} \\
\hline & 1 & 2 & 3 & 4 & 5 & 6 & 7 & \\
\hline Ene & & & 12,5 & 0,05 & 3,3 & 2,6 & 2,5 & 62,9 \\
\hline Feb & & & 1,4 & 0,03 & 4,1 & 0,7 & 2,7 & 69,1 \\
\hline Mar & 8,8 & 1,5 & 13,3 & 2,3 & 5,2 & & 3,6 & 96,9 \\
\hline Abr & 17,2 & 1,9 & 13,2 & 3,0 & 4,0 & & 3,9 & 96,9 \\
\hline May & 26,8 & 2,7 & 13,8 & 3,9 & 3,2 & 0,9 & 4,5 & 101,6 \\
\hline Jun & 11,1 & 3,9 & 13,4 & 4,4 & & 1,7 & 4,4 & 92,4 \\
\hline Jul & & 4,7 & 13,8 & 5,3 & & 0,2 & 4,9 & 59,9 \\
\hline Agt & 8,8 & 3,3 & 1,5 & 0,6 & & 0,8 & 5,1 & 92,4 \\
\hline Sept & 16,7 & 3,1 & 13,1 & 6,5 & & & 4,4 & 81,5 \\
\hline Oct & 25,8 & & 13,4 & 7,4 & & & 4,8 & 81,5 \\
\hline Nov & 10,6 & & 13,1 & 8,0 & & 0,8 & 4,1 & 95,4 \\
\hline Dic & & & 13,1 & 8,7 & 2,1 & 1,7 & 3,8 & 62,9 \\
\hline
\end{tabular}

Tabla 1. Requerimiento hídrico por cultivo a lo largo de un año hidrológicamente seco.

Nota: 1 Habichuela, 2 Maíz, 3 Café, 4 Tomillo, 5 Tomate, 6 Girasol y 7 Pimentón

Al sumar el requerimiento de todos los cultivos se obtiene un caudal de entre $8,91 \mathrm{Lt} / \mathrm{s}$ y $55,82 \mathrm{Lt} / \mathrm{s}$. Restando a estos valores la escorrentía asociada a la precipitación (Qlluvia) se obtiene el caudal teórico de riego, esté último se comparó con la oferta de año seco $\left(\mathrm{Q}_{95}\right)$ y el consumo real del distrito según registros de macromedición.

\begin{tabular}{lrrrcrrr}
\hline Me & Q50 & Q95 & Req & P & Q $_{\text {Il }}$ & Rgo & Mm \\
\cline { 2 - 7 } $\mathrm{s}$ & & $(\mathrm{Lt} / \mathrm{s})$ & & $(\mathrm{mm})$ & & $(\mathrm{Lt} / \mathrm{s})$ & \\
\hline \multirow{2}{*}{ Ene } & 950 & 390 & 20,9 & 15,60 & 3,78 & 17,2 & 46,4 \\
& & & 8 & & & 0 & 9 \\
\hline \multirow{2}{*}{ Feb } & 910 & 340 & 8,91 & 33,20 & 8,85 & 0,06 & 35,2 \\
& & & & & & & 1 \\
\hline \multirow{2}{*}{ Mar } & 111 & 370 & 34,6 & 54,70 & 20,4 & 14,2 & 47,7 \\
& 0 & & 7 & & 5 & 2 & 9 \\
\hline \multirow{2}{*}{ Abr } & 227 & 480 & 43,2 & 105,9 & 39,5 & 3,65 & 32,4 \\
& 0 & & 4 & 0 & 9 & & 2 \\
\hline Ma & 419 & 102 & 55,8 & 125,0 & 49,0 & 6,82 & 14,0 \\
$\mathrm{y}$ & 0 & 0 & 2 & 0 & 0 & & 2 \\
\hline \multirow{2}{*}{ Jun } & 567 & 189 & 38,8 & 117,4 & 41,8 & 0,00 & 5,00 \\
& 0 & 0 & 9 & 0 & 6 & & \\
\hline \multirow{2}{*}{ Jul } & 605 & 190 & 28,8 & 111,1 & 25,6 & 3,20 & 9,48 \\
& 0 & 0 & 6 & 0 & 6 & & \\
\hline \multirow{2}{*}{ Agt } & 503 & 220 & 19,9 & 94,70 & 33,7 & 0,00 & 7,93 \\
& 0 & 0 & 0 & & 6 & & \\
\hline Sep & 327 & 135 & 43,9 & 72,70 & 22,8 & 21,0 & 10,6 \\
$\mathrm{t}$ & 0 & 0 & 1 & & 7 & 4 & 8 \\
\hline
\end{tabular}

\begin{tabular}{lrrrlrrr}
\hline \multirow{2}{*}{ Oct } & 283 & 130 & 51,4 & 84,00 & 26,4 & 25,0 & 13,1 \\
& 0 & 0 & 2 & & 2 & 0 & 7 \\
\hline No & 275 & 121 & 36,6 & 79,40 & 29,2 & 7,44 & 23,0 \\
v & 0 & 0 & 7 & & 3 & & 0 \\
\hline \multirow{2}{*}{ Dic } & 170 & 750 & 29,2 & 35,10 & 8,51 & 20,7 & 26,2 \\
& 0 & & 6 & & & 5 & 6
\end{tabular}

Tabla 2. Calculo de oferta hídrica y riego requerido para un año hidrológicamente seco (Lt/s).

Nota: Req (requerimiento total de agua), Q por precipitación), Rgo (requerimiento para riego), Mm (macromedición)

En la figura 2 se puede observar que la oferta hídrica del río Palmar es suficiente para satisfacer la demanda para riego de ASODAT-ROCA, sin que está última ponga en riesgo la disponibilidad del recurso para otros usos y usuarios. Pude identificarse que entre diciembre y Marzo (periodo de estiaje) se presentan los menores caudales del río Palmar (menor a $500 \mathrm{Lt} / \mathrm{s}$ ), coincidiendo con las altas temperaturas, que pueden llegar a ser de $19,4^{\circ} \mathrm{C}$, es decir, que este es el periodo más exigente para el sostenimiento de los cultivos, no obstante son los meses de septiembre y octubre donde se consume la mayor cantidad de agua para riego $(21,0-25,0 \mathrm{Lt} / \mathrm{s})$.

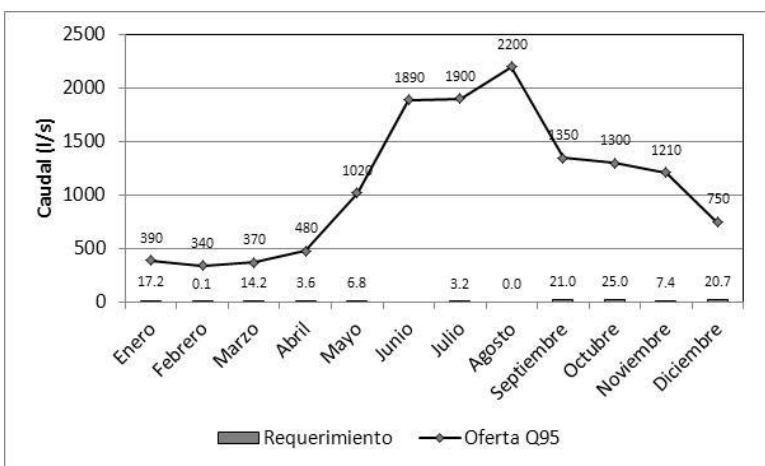

Figura 2. Correlación entre oferta hídrica de año seco Vs requerimiento teórico de riego 


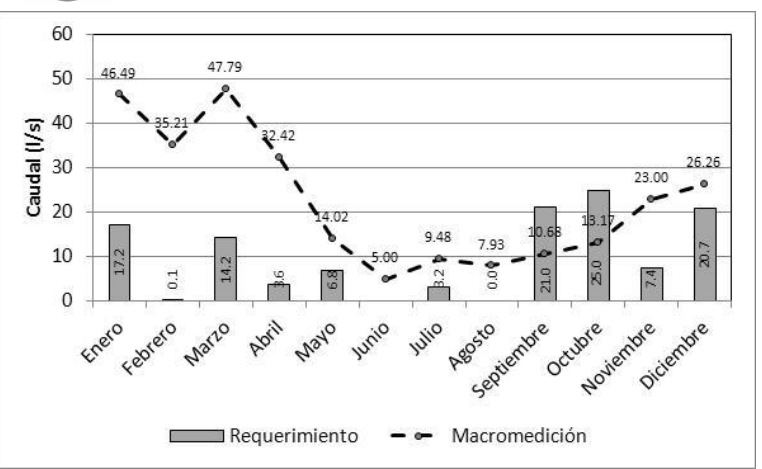

Figura 3. Macromedición Vs requerimiento teórico de riego

La Figura 3 permite inferir que los caudales suministrados por el distrito de riego (macromedición) en ningún mes superan los 60 $\mathrm{Lt} / \mathrm{s}$ otorgados mediante concesión, no está por demás comparar este consumo con el requerimiento teórico de agua para abastecer las condiciones de cada cultivo (obtenido por Blanney Criddle), en cuyo caso se evidencia que entre noviembre y mayo se suministra mayor caudal que el teórico, el cual es considerado en este proyecto como el óptimo, lo que constituye sin duda una oportunidad para formular estrategias que aporten a la sostenibilidad del recurso e incluso al aumento del área irrigable, en tanto ASODAT-ROCA dispone de una concesión de hasta 60 Lt/s. Así mismo, se evidencia que en septiembre y octubre el consumo es inferior al teórico.

\section{Conclusiones}

Se verificó la suficiencia de oferta hídrica del río Palmar para satisfacer la demanda para riego de ASODAT-ROCA, sin arriesgar la disponibilidad del recurso para otros usos y usuarios, incluso en el periodo de estiaje, entre diciembre y marzo, cuando el río tiene menos de 500Lt/s. Sin embargo, según el requerimiento teórico, se podría reducir la presión sobre el recurso e incluso aumentar el área a regar sin que esto signifique un aumento de la concesión.

Los resultados permiten proponer estrategias que complementan los programas tradicionales de un PUEAA, en este caso particular se recomienda: i) realizar mediciones nocturnas del macromedidor para detectar fugas y conexiones ilegales, ii)
63

instalación de una mira para medición de caudales del río Palmar a la altura de la bocatoma para construir una serie histórica de caudales que a futuro permita corroborar la disponibilidad del recurso, iii) instalación de micromedidores en puntos estratégicos de la red de distribución. iv) implementación de sistemas de bajo consumo (riego por goteo) con el fin de asegurar que el agua suministrada llegue directamente a la planta, y v) la adecuación de techos y reservorios para la recolección y aprovechamiento de aguas lluvias.

\section{Referencias Bibliografícas}

Aparicio, F. (1989). Fundamentos de Hidrología Superficial. México: Grupo Noriega Editores.

Organización de las Naciones Unidas para la Agricultura y la Alimentación (2006). Estudio FAO riego y drenaje 56, guías para la determinación de los requerimientos de agua de los cultivos.

IDEAM, \& Ministerio de Ambiente y Desarrollo Sostenible. (2014). Estudio Nacional del Agua. Bogotá D.C.

A. Franco Rojas, Ingeniero Civil de la Universidad Nacional de Colombia; Magister en Recursos Hidráulicos de la Universidad Nacional de Colombia. Docente de la Universidad de La Salle, Facultad de Ingeniería; Integrante activo del Grupo de Investigación en desarrollo tecnológico (INDETEC).M. K. Trochez Páez, Ingeniera Ambiental y Sanitaria de la Universidad de La Salle. Asesora Ambiental Distrito de riego ASODAT-ROCA, Ubaque, Cundinamarca.

*Para citar este artículo:Franco Rojas A; Trochez Páez M.Methodological proposal for the estimation of water supply and demand in a irrigation distric..revista Bistua.2017.15(2):5963

+ Autor para el envió de correspondencia y la solicitud de las separatas: Franco Rojas A. Universidad de La Salle. Email: afrancor@unisalle.edu.co

Recibido: Octubre 30 de 2016

Aceptado: Febrero 04 de 2017 
